\title{
0237 CONSEQUENCES OF MISCLASSIFIED ICD-10 INJURY DIAGNOSES IN A NATIONAL HOSPITAL DISCHARGE REGISTRY
}

M F Bergstrm, L Byberg, H Melhus, K Michaelsson, R Gedeborg* Correspondence: Department of Surgical Sciences, Anaesthesiology and Intensive Care, Uppsala Clinical Research Center, Uppsala University Hospital, UCR/Scheele Dag Hammarskjlds vg 50A, 3tr Science Park SE-751 85 Uppsala, Sweden

\subsection{6/ip.2010.029215.237}

Little is known of the extent and characteristics of primary coding errors and their consequences for epidemiological studies. From the national hospital discharge register we identified 15899 incident injury cases in one region during the years 2000-2004. A random sample of 967 patient records were reviewed. Errors in ICD-10 codes were corrected and the consequences for classification of injuries and external causes, as well as injury severity estimates were analyzed. In $21.4 \%$ of 967 hospital admissions, at least one ICD-10 code for injury was changed or added. Out of 1370 injury codes $10.1 \%$ were corrected. The majority of errors were in the forth position of the code; $94.8 \%$ of the injury codes were correct to the third position. In $10.8 \%$ of the hospital admissions at least one new ICD-10 injury code was added. In the injury matrix classification, $4.6 \%$ of the cases had some category removed and $12.2 \%$ had at least one injury category added. For cases with coding errors, the mean ICD-based injury severity score changed slightly (difference 0.021). The area under the receiver operating characteristic curve was 0.892 for predicting hospital mortality and remained unchanged after corrections of codes. Errors in ICD-10 coded injuries in hospital discharge data are common but most of them are not severe. The consequences for categorisation are moderate and the consequences for injury severity estimates are probably minor. 